\title{
Comparison of methods for static charge energy harvesting on aircraft
}

\author{
M. E. Kiziroglou*a,b,c Th. Becker ${ }^{\mathrm{d}}$, E. M. Yeatman ${ }^{\mathrm{c}}$, U. Schmid ${ }^{\mathrm{e}}$, J. W. Evans ${ }^{\mathrm{a}}$ and P. K. Wright ${ }^{\mathrm{a}}$ \\ ${ }^{a}$ Berkeley Energy and Climate Institute, University of California at Berkeley, CA, 94720, USA \\ ${ }^{\mathrm{b}}$ Department of Automation Engineering T.E., ATEI Thessaloniki, 57400, Greece \\ ${ }^{c}$ Department of Electrical and Electronic Engineering, Imperial College London, SW7 2AZ, U.K. \\ ${ }^{\mathrm{d}}$ Airbus Group Innovations, Communication and Sensor Department, 81663, Munich, Germany \\ ${ }^{\mathrm{e}}$ Institute of Sensor and Actuator Systems, Vienna University of Technology, 1040 Wien, Austria
}

\begin{abstract}
In this paper, the possibility of using the static charge that accumulates on aircraft during flight as a source to power monitoring sensors is examined. The assessed methods include using a pair of materials with different air-flow charging rates, contact discharging of the fuselage to neutral metallic bodies, charge motion induction by the fuselage field and inductive harvesting of fuselage-to-air corona discharges at static discharge wicks. The installation and potential advantages of each method are discussed. The feasibility of directly charging a storage capacitor from accumulated static charge is studied experimentally, demonstrating a voltage of $25 \mathrm{~V}$ on a $25 \mathrm{nF}$ capacitor.
\end{abstract}

Keywords: Energy harvesting, sensors, aircraft, static charge, electrostatic, triboelectricity

\section{INTRODUCTION}

Wireless sensing offers the potential of cost reduction for aircraft operation and maintenance, however, the development and testing of such systems is still limited due to their cabling complications or maintenance overhead due to the requirement for battery replacement. Various technologies have been developing rapidly towards energy autonomous aircraft sensors, mainly using thermoelectric [1], non-inertial piezoelectric [2], or solar [3] systems. In [1], a fully functional wireless strain sensor network with dynamic thermoelectric energy harvesting powered nodes and a custom low-power protocol was demonstrated. The protocol was able to minimize energy consumption by supporting different modes of operation, including deep sleep. The energy harvesting power supplies demonstrated over $100 \mathrm{~J}$ of energy per flight, supporting an $800 \mathrm{~s}$, strain data acquisition and wireless transmission use case. Another harvesting - powered microsystem of interest to avionics, although not specifically developed for aircraft environments is the Michigan Micro Mote ( $\left.\mathrm{M}^{3}\right)$ [4]. This $2 \times 4 \times 4 \mathrm{~mm} 3$ system is integrated with an optical sensor and can transmit data in a range of $2 \mathrm{~m}$, while powered by solar cells providing $0.5 \mu \mathrm{W}$ at $10 \mathrm{kLux}$ (full daylight, not direct sun). A key feature of this system is its $15 \mathrm{nW}$ sleep power. An overview of the state-of-the-art of various energy harvesting technologies, including Wireless Power Transfer (WPT) that may be relevant to aircraft applications is presented in Table 1.

Further to these techniques, the possibility of using the static charge that is accumulated on an aircraft fuselage during flights has been examined [5-7]. However, experimental implementations have been limited, mainly by the difficulty in emulating air-flow induced static charge in laboratory environments. Electrostatic studies of this effect towards the estimation of the corresponding energy availability from within and from the outside of an aircraft structure is also very limited. On the other hand, triboelectric energy harvesting from relative motion between nanostructured surfaces has been studied extensively [8], and impressive simple prototypes have been developed [9]. In this paper, an overview of exploitation methods for static charge developed by relative motion between two solid surfaces is presented. The possibility of energy harvesting from the static charge that builds up on an aircraft fuselage during flight is considered and the main benefits and challenges are discussed.

*m.kiziroglou@berkeley.edu

Smart Sensors, Actuators, and MEMS VIII, edited by Luis Fonseca, Mika Prunnila, Erwin Peiner, Proc. of SPIE Vol. 10246, 102460X - (C) 2017 SPIE · CCC code: 0277-786X/17/\$18 · doi: 10.1117/12.2264890 


\begin{tabular}{|c|c|c|}
\hline Powering Source & Power Density State-Of-Art & Indicative Aircraft Application \\
\hline Direct thermoelectric & $\begin{array}{l}0.5 \mathrm{~mW} / \mathrm{cm}^{2} @ \Delta \mathrm{T}=5 \mathrm{~K} \\
10 \mathrm{~mW} / \mathrm{cm}^{2} @ \Delta \mathrm{T}=20 \mathrm{~K}[10]\end{array}$ & Aircraft seat sensors [11] \\
\hline Dynamic thermoelectric & $\begin{array}{l}1 \mathrm{~mW} / \mathrm{g} \text { in } 2 \text { hour flight } \\
1 \mu \mathrm{W} / \mathrm{g}, 24 \text { hour day/night cycle [1] }\end{array}$ & Fuselage strain sensors \\
\hline Outdoor solar & $\begin{array}{l}10 \mathrm{~mW} / \mathrm{cm}^{2} @ \text { direct sun }(\eta=10 \%) \\
2 \mathrm{~mW} / \mathrm{cm}^{2} \text { daily average }(5.5 \mathrm{~h} / \text { day })\end{array}$ & Exterior sensors \\
\hline Indoor solar & $\begin{array}{l}10 \mu \mathrm{W} / \mathrm{cm}^{2}, \text { diffused } 400 \mathrm{~lm} \\
\text { (internal data) }\end{array}$ & Low duty-cycle cabin sensors \\
\hline Airflow & $4 \mathrm{~mW} / \mathrm{cm}^{2} @ 10 \mathrm{~m} / \mathrm{s}[12]$ & Ventilation area sensors \\
\hline Motion & $\begin{array}{l}0.1 \mathrm{~mW} / \mathrm{cm}^{3}, 100 \mathrm{~Hz}[13] \\
10 \mu \mathrm{W} / \mathrm{g}, 700 \mathrm{~Hz} \text { (internal data) }\end{array}$ & Helicopter motor tuned harvesting \\
\hline Inductive WPT & $1 \mathrm{~mW} @$ range = 5×size [14] & Passenger Service Unit sensors \\
\hline Acoustic WPT & 1 mW/cm³ @1 m of metal [15] & Fuselage strain sensors \\
\hline
\end{tabular}

Table 1. State-of-the-art of power availability from various energy harvesting sources and indicative aircraft sensor applications.

\section{HARVESTING FROM RELATIVE MOTION BETWEEN SURFACES}

The main possible mechanisms of charge transfer for static charge induced by relative motion between two insulating surfaces in contact are illustrated in Figure 1 The relative motion results in repeating contact and separation between microscale areas of the surfaces, which result in a net total charge exchange, usually due to the difference between the energy of electrons in the two materials near the Fermi level. This results in space charge near the surface of the insulating materials. This space charge could then be exploited by channeling the charge to an electrical circuit comprising a storage unit such as a capacitor, through electrodes that can be built in the insulation material. This case is depicted in Figure 1 (top). The local charge diffusion through the insulating area towards an electrode is slow and can be impeded by the presence of the neighboring opposite charge in the opposite material. The design of such embedded electrodes should maximize the covered area, for a given charge path distance, e.g. by minimizing the thickness of the insulating triboelectric material and prevent (or account for) the possibility of contact between opposing electrodes during motion.

Another way to collect static charge developed on a surface by triboelectricity is by scanning the charged insulating area with an electrode. This case is illustrated in Figure 1 (middle). In this case, the trapped charge is exploited in two different ways. The electrode can pick up a small portion of the charge that is trapped on the surface, but at the same time, charge in the electrode is re-distributed as it moves within the electric field of the static charge. Thereby, a current flow is induced into the electrode.

This method has been experimentally studied, in terms of its ability to store electrical energy into a capacitor. For the experiment, an acetate sheet was used as the insulator. The acetate sheet was triboelectrically charged by rubbing with a polyester cloth. Then, the charged area was scanned, using a smaller $\mathrm{Cu}$ sheet as an electrode. The $\mathrm{Cu}$ electrode was connected to a $25 \mathrm{nF}$ ceramic capacitor. The other electrode of the capacitor was connected to an aluminum plate which was placed underneath the acetate sheet, serving as a ground plane. Subsequently, the capacitor was connected to a $1 \mathrm{M} \Omega$ input resistance oscilloscope channel, to measure the voltage. A typical capacitor discharge was observed, as shown in Figure 2 (left), corresponding to the time constant of the system and demonstrating a voltage of $25 \mathrm{~V}$ across the capacitor. 
The equivalent circuit of the measurement setup is shown in Figure 2 (right). This corresponds to a stored energy of around $8 \mu \mathrm{J}$. The main disadvantage of this operation principle is the requirement of two motion stages, one for charge development and one for charge collection. However, a mechanical design could combine these operational motion stages by integrating the conductive electrode into the moving triboelectric surface, in order to exploit both space-charge collection and inductive operation. In order to characterise the performance of such devices, which involve capacitances in the femto-Farad range, suitable high input resistance measuring circuits can be employed [16].

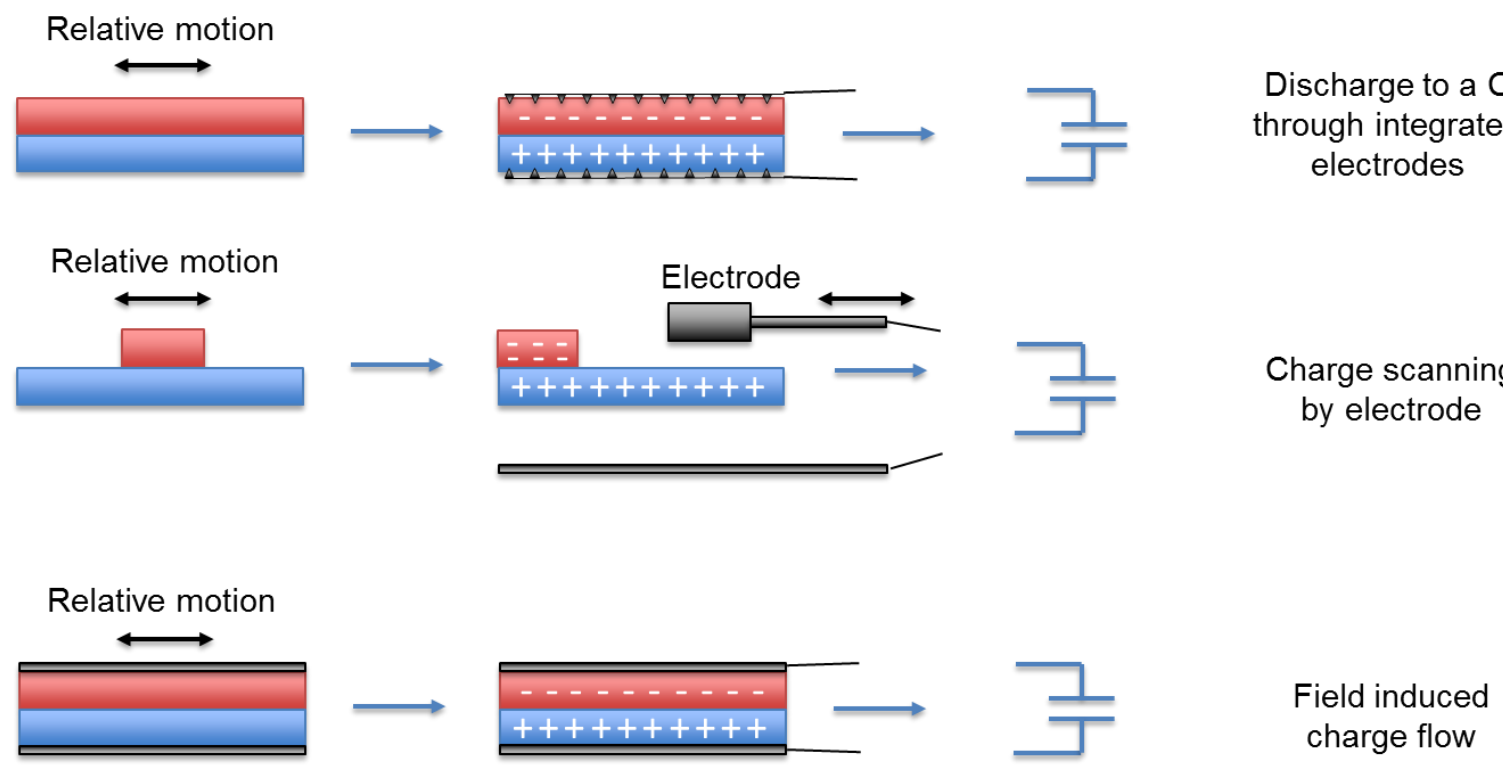

Figure 1. Charge transfer methods for static charge induced by relative motion between two surfaces in contact. Top: Discharge through integrated electrodes. Middle: Charge scanning using an electrode. Bottom: Electrostatic field induction. A very weak field on the outer side of the charged insulating materials is expected.

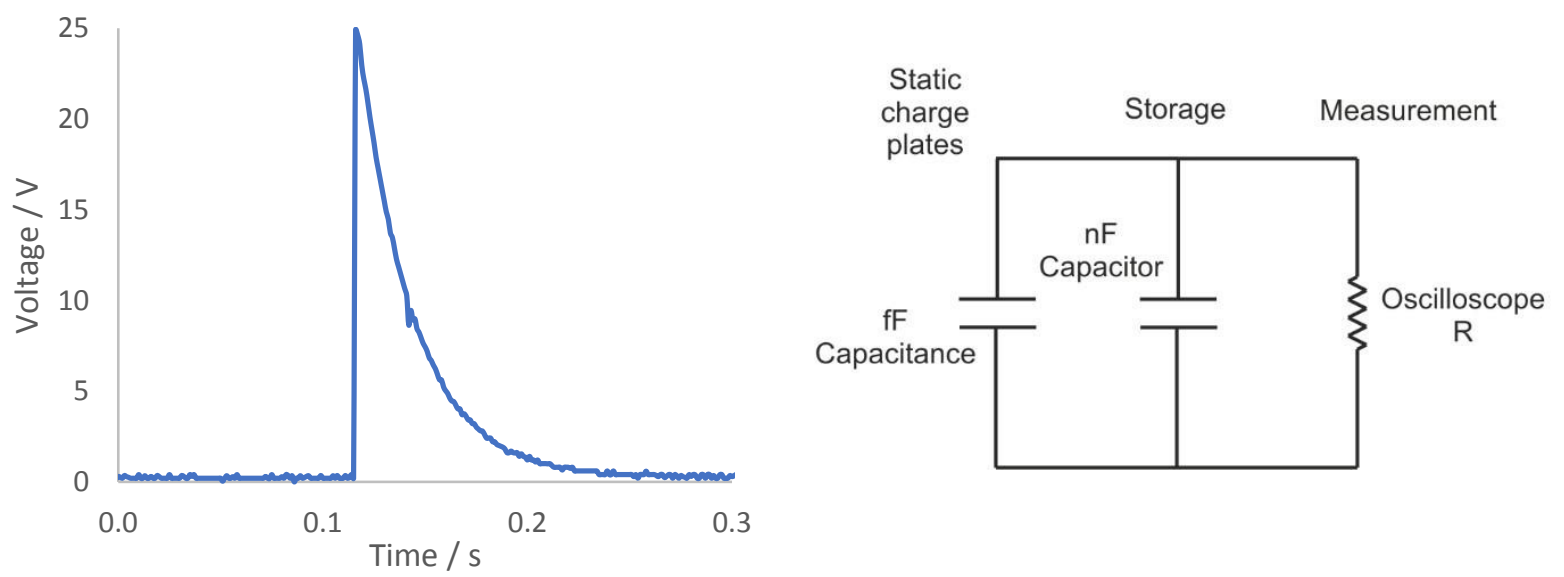

Figure 2: Left: Static charge on a $25 \mathrm{nF}$ capacitor accumulated by surface scanning as shown in the middle schematic of Figure 1. When connected, the $25 \mathrm{~V}$ voltage is discharging into the $1 \mathrm{M} \Omega$ input resistance of the oscilloscope used for the measurement. The corresponding energy is $8 \mu \mathrm{J}$. Right: The circuit equivalent of the system during the measurement. 
A third possible method of static charge exploitation could involve the employment of conductive layers at the back of the triboelectric surfaces, as shown at the bottom of Figure 1. This could also combine charge pick up and induction, during the triboelectric static charge build-up. However, the distance between the static charge and the electrodes is larger meaning that charge pick-up may be limited, even in the case of thin triboelectric materials. In addition, the induction effect may also be limited, because the electric field is expected to be directed inwards, towards the interface between the oppositely charged insulators.

\section{UTILIZATION OF THE FUSELAGE STATIC CHARGE}

The case of airflow-induced, aircraft fuselage static charge and possible charge transfer methods are shown in Figure 3. This charge can be exploited through the electric field around the surface of the fuselage. If a capacitor structure is placed in close vicinity to the fuselage surface, the increasing electric field could polarize the dielectric material and induce an increasing voltage between two conductive plates (Figure 3, top). Alternatively, the fuselage itself could be used as one of the capacitor plates. The energy stored in this capacitive structure could then be used to power an electronic system [5] .

The exploitation of the electrostatic field of the fuselage as an energy source for aircraft sensors has also been proposed in [17]. In that method, the electrostatic force is used to create repeatable motion on a cantilever, e.g. by repetitive charging (or discharging) through electrical contact between the fuselage and another conductive section. This conductive section could be a separate conducting body at the aircraft interior, a separate fuselage surface with different air interfacing or a discharge wick tip. Then, electrical energy can be collected either by exploitation of the resulting discharge pulses, or by impeding the mechanical motion with a piezoelectric or electromagnetic transduction mechanism.

Furthermore, static charge accumulation could potentially be utilized by inductive exploitation of corona discharges to the air environment at discharge wicks or rods. A schematic illustration of this approach is shown in Figure 3 (bottom). Discharge wicks provide a high resistance discharge path between the fuselage and the surrounding air, by implementing a tip geometry that increases the strength of the electrostatic field. In this way, corona discharge is directed to the wicks and controlled to provide a smooth leakage to the atmosphere. This is critically important to minimizing corona-induced $\mathrm{RF}$ interference to the aircraft communication channels. This leakage current could potentially be exploited by an inductive or other mechanism to implement self-powered aircraft sensors in the vicinity of discharge wicks.

Fuselage outer surface

$+++++++++++++++++++++++++++++++$

Fuselage inner surface
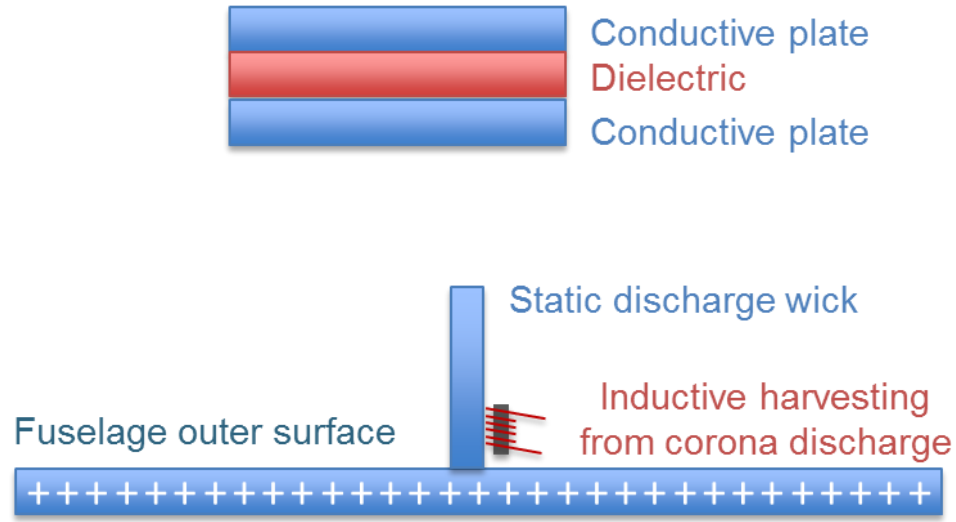

Figure 3: Top: Field-effect / inductive charging of a capacitive structure near the fuselage. The field will be stronger on the outside surface. Bottom: Inductive harvesting from corona discharges to air using discharge wicks. 


\section{CONCLUSIONS}

Various methods for collecting static charges at aircraft surfaces and their technical implementations have been presented. These methods, in general, exhibit promising characteristics for powering future wireless sensors in aircraft. The presented results demonstrate that static charge can be collected and stored in a capacitor component. In the particular experimental results presented here, a $25 \mathrm{nF}$ ceramic capacitor was charged at $25 \mathrm{~V}$ using a $2 \times 3 \mathrm{~cm}$ moving electrode. The corresponding stored harvested energy is $8 \mu \mathrm{J}$. It is concluded that static charge energy can be stored in a usable form and at convenient voltage levels, provided that a motion system that combines static charge generation and charge collection is being used. Using moving electrodes may also be beneficial for the exploitation of static charge building up on the fuselage during flight, but employing electrostatic generators that take advantage of the fuselage electrostatic field or electrostatically driven cantilever motion with electromagnetic or piezoelectric transducers. Finally, static charge leakage through fuselage discharge wicks and rods could potentially be used for powering sensors in their vicinity. However, further investigations are needed in order to assess the energy availability, efficiency and density from static charge energy harvesting devices and the installation opportunities at aircraft fuselage surfaces.

\section{REFERENCES}

[1] M. E. Kiziroglou, S. W. Wright, T. T. Toh, P. D. Mitcheson, T. Becker, and E. M. Yeatman, "Design and Fabrication of Heat Storage Thermoelectric Harvesting Devices," Industrial Electronics, IEEE Transactions on, vol. 61, no. 1, pp. 302-309, 2014.

[2] S. W. Arms et al., "Energy harvesting wireless sensors and networked timing synchronization for aircraft structural health monitoring," in Wireless Communication, Vehicular Technology, Information Theory and Aerospace \& Electronic Systems Technology, 2009. Wireless VITAE 2009. 1st International Conference on, 2009, pp. 16-20.

[3] K. C. Magoteaux, B. Sanders, and H. A. Sodano, "Investigation of an energy harvesting small unmanned air vehicle - art. no. 692823," in Active and Passive Smart Structures and Integrated Systems 2008, vol. 6928(Proceedings of the Society of Photo-Optical Instrumentation Engineers (Spie), 2008, pp. 92823-92823.

[4] G. Kim et al., "A millimeter-scale wireless imaging system with continuous motion detection and energy harvesting," in 2014 Symposium on VLSI Circuits Digest of Technical Papers, 2014, pp. 1-2.

[5] T. Becker, A. Elefsiniotis, and U. Schmid, "Energy harvester, aircraft component comprising the energy harvester and an aircraft comprising the energy harvester or the aircraft component," Patent, Espacenet, EP2886464 (A1), 2015.

[6] H. Xie, Z. Huang, S. Guo, and E. Torru, "Feasibility of an Electrostatic Energy Harvesting Device for CFCs Aircraft," Procedia Engineering, vol. 99, pp. 1213-1222, 2015/01/01 2015.

[7] P. O. Jarvinen, "P-static energy source for an aircraft," Patent, 7,592,783 B1, USA, 2009.

[8] Z. L. Wang, L. Lin, J. Chen, S. Niu, and Y. Zi, "Triboelectric Nanogenerators," in Triboelectric NanogeneratorsCham: Springer International Publishing, 2016, pp. 1-517.

[9] M. E. Karagozler, I. Poupyrev, G. K. Fedder, and Y. Suzuki, "Paper generators: harvesting energy from touching, rubbing and sliding," presented at the Proceedings of the 26th annual ACM symposium on User interface software and technology, St. Andrews, Scotland, United Kingdom, 2013.

[10] M. E. Kiziroglou et al., "Scaling and super-cooling in heat storage harvesting devices," Microsystem Technologies, journal article vol. 22, no. 7, pp. 1905-1914, 2016. 
[11] D. Samson, M. Kluge, T. Becker, and U. Schmid, "Energy Harvesting for Remote Monitoring of Aircraft Seats," Sensor Letters, vol. 8, no. 2, pp. 328-335, Apr 2010.

[12] D. A. Howey, A. Bansal, and A. S. Holmes, "Design and performance of a centimetre-scale shrouded wind turbine for energy harvesting," Smart Materials and Structures, vol. 20, no. 8, p. 085021, 2011.

[13] A. C. Waterbury and P. K. Wright, "Vibration energy harvesting to power condition monitoring sensors for industrial and manufacturing equipment," Proceedings of the Institution of Mechanical Engineers, Part C: Journal of Mechanical Engineering Science, vol. 227, no. 6, pp. 1187-1202, 2013/06/01 2012.

[14] D. E. Boyle, M. E. Kiziroglou, P. D. Mitcheson, and E. M. Yeatman, "Energy Provision and Storage for Pervasive Computing," IEEE Pervasive Computing, vol. 15, no. 4, pp. 28-35, 2016.

[15] M. E. Kiziroglou, D. E. Boyle, S. W. Wright, and E. M. Yeatman, "Acoustic power delivery to pipeline monitoring wireless sensors," Ultrasonics, vol. 77, pp. 54-60, 5// 2017.

[16] M. E. Kiziroglou, C. He, and E. M. Yeatman, "Rolling Rod Electrostatic Microgenerator," IEEE Transactions on Industrial Electronics, vol. 56, no. 4, pp. 1101-1108, Apr 2009.

[17] A. Elefsiniotis, T. Becker, and U. Schmid, "Resonant energy harvester and an aircraft comprising the resonant energy harvester," Patent, Espacenet, EP2887531 B1, 2015. 